M. Šejnoha, et al., Int. J. Comp. Meth. and Exp. Meas., Vol. 7, No. 2 (2019) 167-180

\title{
EFFECTIVE MATERIAL PROPERTIES OF WOOD BASED ON HOMOGENIZATION
}

\author{
M. ŠEJNOHA ${ }^{1}$, L. KUCÍKOVÁ ${ }^{1}$, J. VOREL ${ }^{1}$, J. SÝKORA ${ }^{1} \&$ W.P. DE WILDE ${ }^{2,3}$ \\ ${ }^{1}$ Czech Technical University in Prague, Faculty of Civil Engineering, Department of Mechanics. \\ ${ }^{2}$ Vrije Uiniversiteit Brussel. \\ ${ }^{3}$ Wessex Institute of Technology.
}

\begin{abstract}
This paper is concerned with the evaluation of effective material properties of wood. Since both mechanical loading and climatic changes play a crucial role in the prediction of wood response, we consider not only stiffness, but also non-mechanical properties driving the heat and moisture transport and thus indirectly addressing the swelling and shrinkage properties of wood. In this regard, classical micromechanical models as well as numerical simulations based on the Extended Finite Element Method are examined. A special attention is devoted to the influence of microstructural details of the porous phase. To that end, the X-ray computational micro-tomography is adopted when seeking for information beyond the volume fraction of phases that can be identified at various levels of a hierarchical arrangement of wood. A spruce wood is selected as one particular example to compare individual computational approaches.

Keywords: Conductivity, diffusivity, homogenization, microfibril angle, micro-tomography, Mori-Tanaka method, nanoindentation, stiffness, X-FEM.
\end{abstract}

\section{INTRODUCTION}

Timber structures have been recognized as a promising alternative to traditional steel/concrete/ masonry construction approaches. The glued laminated timber (GLT) beams in particular have enjoyed a particular attention. These are typically of higher grade in comparison to solid timber and thus less sensitive to local defects being the source of damage evolution. While mechanical loading is certainly the most severe load contribution, the climatic changes, the wood structures are often exposed to, may considerably influence the structure durability and thus its service life [1, 2]. A rigorous fully coupled hydro-thermo-mechanical model capable of accounting for moisture induced stresses is therefore of paramount importance. This has been confirmed in [3] clearly demonstrating the mechanosorption effects investigated experimentally at the level of annual rings during creep experiments through the measurements of local strains using the grid method. Similar works allowing for full field measurements of strain profiles, although limited to free swelling caused by moisture uptake, were reported in $[4,5]$ exploiting the digital image correlation method.

For such a complex model to provide reliable predictions at structural level, the proper determination of material parameters at this level of observation is doubtless one of the most important steps. This issue gains in complexity if accepting the natural variability of wood properties. Regarding the structural behavior, this issue has been addressed, e.g. in [6, 7]. Much effort has also been devoted to the effect of microstructural details. The determination of variability of microfibril angle (MFA) has been studied in $[8,9]$ following the steps set in [10]. It has been observed that MFA not only reduces the wood stiffness, but also increases shrinkage [11], which inherently depends on the variation of moisture content in wood. While hierarchical arrangement of wood has been addressed in number of contributions to arrive at effective transport properties such as thermal conductivities [12] and moisture diffusivities [13] the influence of MFA variation has been examined in the light of elastic stiffness 
properties so far. The analytical micromechanics models such as the Mori-Tanaka or Self-consistent schemes have been adopted to arrive at effective properties [14, 15] thus limiting the attention to volume fraction and shape and orientation of inclusions. On mesoscale (the level of earlywood and latewood) as well as on macro-scale (the level of annual rings) the former variable is often related to the wood density [16]. Although the phase volume fraction seems pivotal in the prediction of effective properties, the number of contributions attempting to account for more detailed morphological description of wood microstructure is scarce. Some preliminary studies that promote the use of computational micro-tomography can be found in [17].

Some of the previous issues are revisited in this paper with emphases on the effect of microstructural details. Attention is limited to steady state or quasi-static analysis. Section 2 addresses some of the microstructure related parameters such as the MFA, phase volume fractions, and geometrical details of the porous phase. The adopted computational methods are shortly described next in Section 3. The homogenized properties are summarized in Section 4 for the spruce wood as the most typical type of wood used in the construction of GLT beams in the Czech Republic. The most important concluding remarks are finally provided in Section 5.

\section{WOOD MICROSTRUCTURE}

The literature offers a number of contributions being concerned with a highly irregular microstructure of wood, which progresses across several scales [11, 16, 15, to cite a few]. Three such scales can be identified in Fig. 1(a) making also distinction between earlywood and latewood. They differ not only in the volume of lumens (the hollow tubes surrounded by the cell wall material), but also in their geometrical characteristics, see Figs. 1(b,c). ${ }^{1}$ Further details can be found in the previously cited literature.

\subsection{Microfibril angle}

If zooming in to the cell wall displayed in Fig. 1(d) one may recognize several sequentially deposited layers building up the wall. About $80-90 \%$ of the total cell wall thickness is taken by the secondary layer (S2) $[16,18]$, which is the major contributor to the mechanical properties of wood cell walls. The material species found within individual layers can be assumed tissue independent, universal to all woods. The cell wall microstructure thus invites additional downscaling as adopted in $[16,15]$. Among all species the crystalline cellulose deserves a particular attention. This is because the orientation of microfibrils of the crystalline

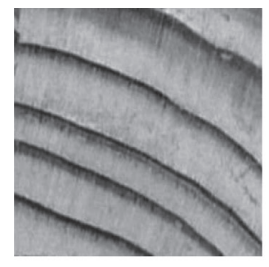

(a)

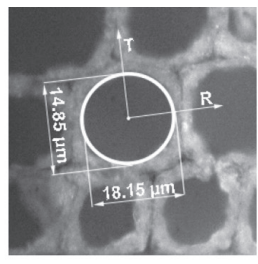

(b)

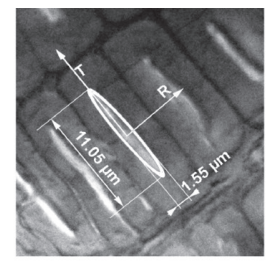

(c)

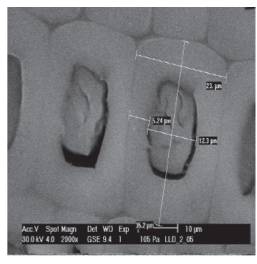

(d)

Figure 1: Hierarchical arrangement of softwood (spruce): (a) annual rings, (b) early-wood, (c) latewood, (d) cell wall.

\footnotetext{
${ }^{1}$ Material axes in the transverse plane represent the radial $(\mathrm{R})$ and tangential $(\mathrm{T})$ direction.
} 
cellulose, or rather their deviation from the direction of lumens, considerably influences the cell wall stiffness $[18,15]$ and other wood properties such as shrinkage or swelling [11].

In this regard, a direct estimation of MFA from image processing can successfully be substituted by indirect measurements using indentation combined with homogenization. This appears much advantageous as nanoindentation combined with homogenization at the cell wall level or macroindentation using the Pilodyn device, where homogenization across all scales is needed, allows us to obtain the whole transversely isotropic stiffness matrix of the cell wall material. For further details the interested reader is referred to $[19,10,6,8,9]$. For illustration we plot in Fig. 2(a,b) the probability distribution functions of indentation modulus obtained from two samples of spruce and the corresponding MFA derived from the application of anisotropic theory of nanoindentation [20,21] to advocate a random character of MFA. How severely the stiffness properties are dependent on MFA is evident from Fig. 2(c).

\subsection{Volume fraction of wood on various scales}

The volume fractions of individual components entering the micro-scale (cell wall level) homogenization step can be identified from their weight fractions and apparent densities as presented in [16] for a large group of wood plants. Similar procedure can be adopted for the derivation of the volume fraction of pores (lumens) of earlywood $\left(c_{L E}\right)$ and latewood $\left(c_{L L}\right)$ as well as the volume fractions of earlywood $\left(c_{E W}\right)$ and latewood $\left(c_{L W}\right)$.

Another option is to employ an image analysis to acquire the volume fractions from two-dimensional binary images of a real microstructure. An illustrative example pertinent to the earlywood of spruce is presented in Fig. 3. The results obtained from the two approaches

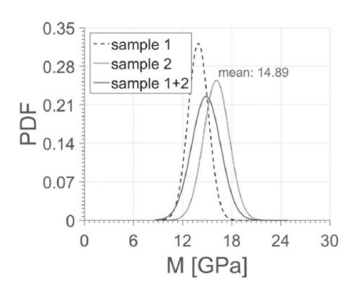

(a)

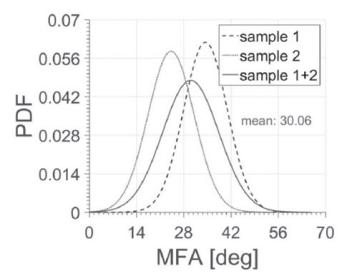

(b)

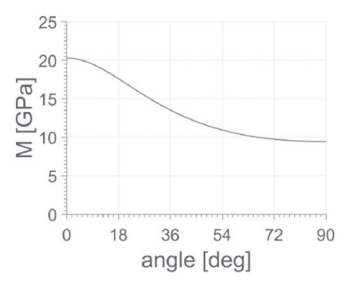

(c)

Figure 2: Probability distribution function of: (a) indentation modulus, (b) MFA; (c) variation of indentation modulus as a function of MFA.

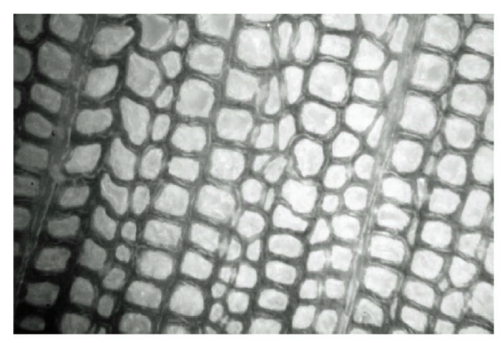

(a)

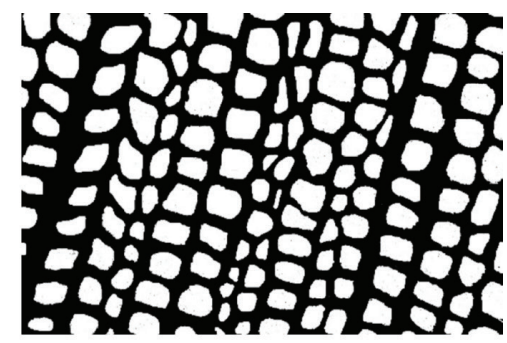

(b)

Figure 3: Images of earlywood of spruce: (a) original grayscale image, (b) transformed binary image. 


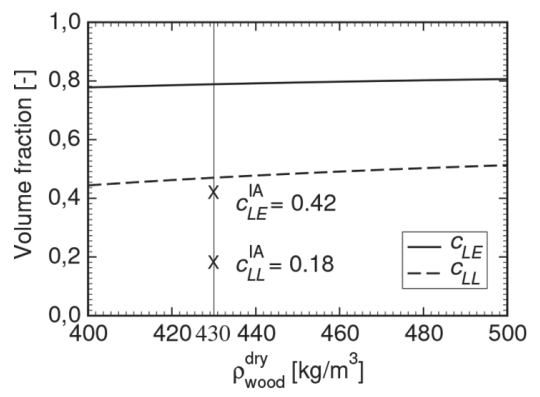

(a)

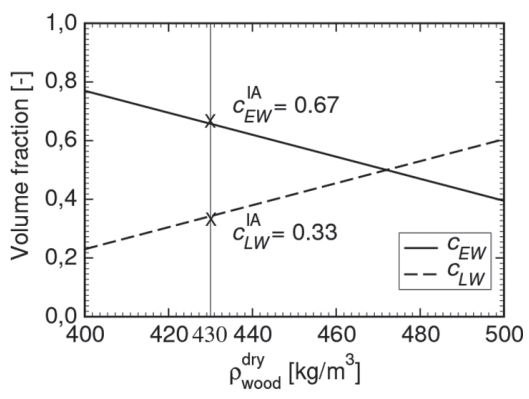

(b)

Figure 4: Volume fractions plotted as a function of dry wood density: (a) volume fractions of lumens, (b) volume fractions of earlywood and latewood.

are then compared in Fig. $4 .^{2}$ This opens some questions as to the reliability of the prediction of effective properties if not addressing the microstructure with its all details. This issue is partially explored in the next section.

\subsection{X-ray computational microtomography}

Much higher degree of information about the wood microstructure and thus also about the degree of porosity can be obtained with the help of computational micro-tomography ( $\mu \mathrm{CT}$ ). The stepping stone in the construction of a suitable computational model (representative volume element, RVE) from the voxel structure of the reconstructed 3D image containing the entire grayscale. Such image must therefore be segmented to computationally identify the structure and volume of individual phases.

A suitable method of attack is the procedure based on the grayscale histogram thresholding using hierarchical cluster analysis [22]. This method can be briefly characterized as follows. It is assumed that the target histogram contains $N$ different nonempty gray levels which can be grouped into several upwardly ordered clusters $C_{k}, k=1, \ldots, K$. At the beginning of the segmentation process each cluster accommodates only one gray level $(K=N)$. A certain measure of dissimilarity of two adjacent clusters $C_{i}, C_{j}$, termed the cluster distance in [22], is then evaluated as

$$
\operatorname{Distance}\left(C_{i}, C_{j}\right)=\sigma_{I}^{2}\left(C_{i} \cup C_{j}\right) \sigma_{A}^{2}\left(C_{i} \cup C_{j}\right),
$$

where the so called inter-class variance $\sigma_{\mathrm{I}}^{2}\left(C_{i} \cup C_{j}\right)$ is defined as the sum of the square distances between the means of the two clusters and the total mean of both clusters and is $\sigma_{A}^{2}\left(C_{\mathrm{i}} \cup C_{j}\right)$ defined as the variance of all pixel values in the merged cluster, see [22] for their particular forms. The pair of clusters with the smallest distance is then merged. This reduces the number of clusters and has to be repeated $N-M$ times to obtain $M$ desired clusters.

A graphical representation of the above smoothing process applied to the sample of earlywood of spruce is displayed in Fig. 5. Unfortunately, this approach crucially depends on the quality and spacial resolution of the starting image. If the noise is too high or the resolution

\footnotetext{
2 The values corresponding to image analysis are labeled with superscript (IA). These were found for wood initially dried in the oven at $60^{\circ} \mathrm{C}$ to correspond to values calculated from dry wood density $\rho_{\text {wood }}^{\text {dry }}$
} 


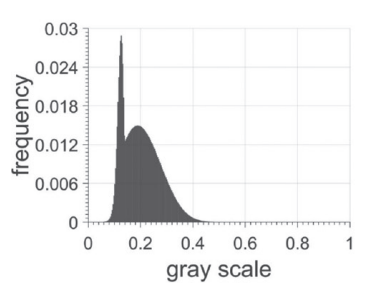

(a)

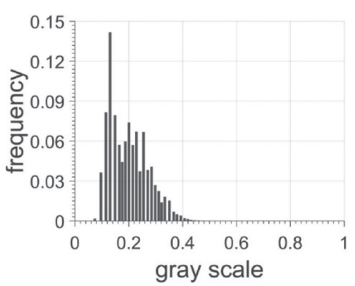

(b)

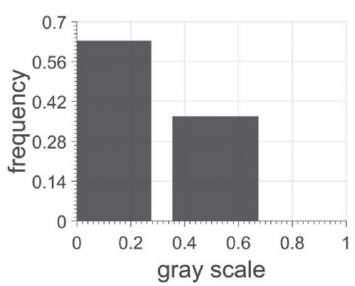

(c)

Figure 5: Histograms of the grayscale for the selected number of clusters during the segmentation process: (a) 201, (b) 43, (c) 2 .

and contrast too low then only some feature descriptors can be determined. The main difficulty lies in distinguishing the objects (defects) from one another and the correct determination of material interfaces.

This obstacle has been overcome by repeating this process twice taking advantage of the available single source double-energy CT scans [23]. First, the reconstructed images for two energy levels (low $(L E)$ and high $(H E)$ ) are segmented based on the described hierarchical clustering. The material is unambiguously determined for pixels where both segmentations give the same results. The material of remaining pixels is determined based on the segmentation of the weighted summation of a set of spectra $\Omega=c_{L E} \Omega_{L E}+c_{H E} \Omega_{H E}$, where $c_{L E}$ and $c_{H E}$ are the weights of respected CT scans $\left(c_{L E}+c_{H E}=1, c_{L E}, c_{H E}>0\right)$. These coefficients are fitted to minimize the error between the resulting combined spectra histogram and the spectra histograms for given energy levels.

The resulting reconstructed images distinguishing between cell wall material and the structure of lumens are shown in Fig. 6. The volume fractions rendered by individual approaches are listed in Table 1. Clearly, the volume fraction of earlywood provided by the

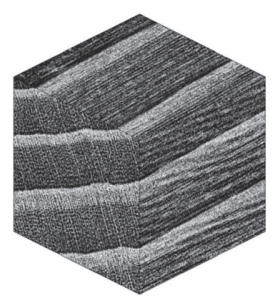

(a)

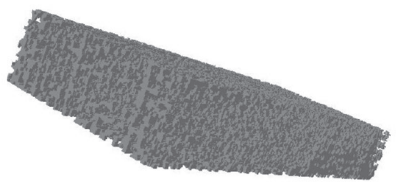

(b)

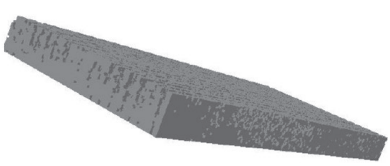

(c)

Figure 6: Reconstructed 3D images from single source double-energy method: (a) annual rings, (b) earlywood, (c) latewood.

Table 1: Volume fractions of earlywood and latewood from individual approaches.

\begin{tabular}{lcc}
\hline Method & Earlywood & Latewood \\
\hline Wood density & 0.78 & 0.47 \\
2D image analysis & 0.42 & 0.18 \\
$\mu$ CT dual-energy & 0.75 & 0.20 \\
\hline
\end{tabular}


2D image analysis is highly underestimated. Furthermore, owing to the difficulty associated with the determination of volume fraction of latewood from available CT-scans and remembering, on the contrary, a good match for earlywood, we may support the determination of the volume fraction from the wood density with sufficient reliability. From a different prospective, a variability of results from individual approaches suggests treating the volume of pores as a random variable. Such a viewpoint fits well within the concept of parameter identification based on the Bayesian inference, see e.g. [9] for more details.

\section{COMPUTATIONAL APPROACH}

The derivation of homogenization (effective) material properties relies in general on two types of approaches. One based on the solution of the Eshelby transformation inclusion problem and one based on the definition of a suitable computational model in the form of a periodically repeating RVE, which is typically analyzed numerically with the help of the finite element method. The first approach is usually promoted when no details about the local fields on the scale of individual constituents is needed. This is generally the case, examined also in this paper, when only the effective properties, to be used in an independent macroscopic analysis, are required. Henceforth, the two approaches are therefore compared only in the light of the applied microstructural details.

\subsection{Mori-Tanaka method}

The Mori-Tanaka (MT) method belongs to the class of the average field models where the Eshelby problem is understood such that a single inclusion is embedded into homogeneous unconstrained matrix and loaded in the infinity by the macroscopic fields [14, 15]. To avoid potential drawbacks of these analytical models delivering non-symmetric stiffness matrices in case of multi-phase material systems [24] we limit our attention in all homogenization problems two phase materials only. In the spirit of the Mori-Tanaka method the effective properties on a given scale are provided by

$$
\mathbf{L}^{\text {hom }}=\mathbf{L}_{1}+c_{2}\left(\mathbf{L}_{2}-\mathbf{L}_{1}\right) \mathbf{A}_{2}
$$

where subscript 1 is typically reserved for the matrix phase and subscript 2 for the inclusion. The matrix $\mathbf{A}_{2}(6 \times 6$ in case of elasticity, $3 \times 3$ in case of moisture diffusion or heat conduction) represents the concentration or localization factor depending on the material properties of both phases and shape and orientation of the inclusion. The matrices $\mathbf{L}_{1}, \mathbf{L}_{2}$ store material properties of individual phases, i.e. $\mathbf{L}_{i}$ is either the $6 \times 6$ stiffness matrix, $3 \times 3$ diffusivity matrix or $3 \times 3$ conductivity matrix of a given phase $i$.

Point out that the MT method is, unlike the Self-consistent method, fully explicit and boils down, providing the localization matrix $\mathbf{A}_{i}$ is known, to simple matrix multiplication. Closed form solutions for several types of ellipsoidal inclusions (spheres, cylinders, ellipsoids) are available, e.g. [15]. In comparison to numerical analysis carried out on a given RVE the MT method is therefore computationally much more efficient.

While eqn (2) is generally applicable to the level of cell wall and the level of lumens, a different homogenization strategy is needed for the level of annual rings. The most simple approach accepts the laminate like structure of annual rings to reduce the last homogenization step to simple Voigt and Reuss bounds, see e.g. [15]. Herein, this step was utilized within the Mori-Tanaka method only to get the results presented in Fig 8. 
As also presented in $[16,12,25]$ the prediction of effective elastic stiffnesses and thermal conductivities requires two additional homogenization steps at the cell wall level in comparison to effective diffusivities [13].

\subsection{Extended finite element method}

When microstructure details beyond the phase volume fractions are available, or the distribution of local fields is needed, the numerical homogenization is usually selected. In most cases the 1 st order homogenization theory [26, 27, 28], which assumes constant macroscopic gradients acting over a sufficiently large volume of macroscopically homogeneous material, is given the preference over more complex approaches [29]. On a lower scale, such volume is typically represented by a periodically repeating RVE being representative to actual microstructure as close as possible. To that end, either statistically periodic unit cells [30] or reconstructed $\mu \mathrm{CT}$ images [31] are then employed. Hereafter, the latter approach is exploited.

To begin, we split the distribution of local fields over RVE (displacements $\boldsymbol{u}$, temperature $\theta$, moisture $\varphi$ ) into a homogeneous part, linearly varying over RVE, and a fluctuation part reflecting the effect of heterogeneities as ${ }^{3}$

$$
\boldsymbol{u}=\boldsymbol{E} \cdot \boldsymbol{u}+\boldsymbol{u}^{*}, \boldsymbol{\varepsilon}=\boldsymbol{E}+\boldsymbol{\varepsilon}^{*}, \boldsymbol{\sigma}=\mathbf{L}\left(\boldsymbol{E}+\boldsymbol{\varepsilon}^{*}\right),
$$

where $\boldsymbol{E}$ is the macroscopically homogeneous strain and $\boldsymbol{u}^{*}$ store the periodic displacement fluctuations, $\boldsymbol{\varepsilon}^{*}$ is the corresponding strain, and the local stress $\boldsymbol{\sigma}$ follows from Hooke's law written in terms of phase stiffness matrix $\mathbf{L}$ and the local strain. Next, substituting eqn (3) into the principal of virtual work (Hill's lemma) and assuming the strain loading conditions $(\delta \boldsymbol{E}=\mathbf{0})$ gives

$$
\left\langle\delta \boldsymbol{\varepsilon}^{* \mathrm{~T}} \mathbf{L} \boldsymbol{\varepsilon}^{*}\right\rangle=-\left\langle\delta \boldsymbol{\varepsilon}^{* \mathrm{~T}} \mathbf{L} \boldsymbol{E}\right\rangle,
$$

where \langle\rangle stands for the volume averaging. Equation (4) is to be solved numerically. To avoid difficulties with meshing complex microstructures in Fig 6 using standard finite element formulation we turn our attention to work of Moës et al. [32] and employ the approach based on the Extended Finite Element Method (X-FEM). Because X-FEM enables an application of regular meshes, e.g. standard brick elements, which do not have to confirm to physical boundaries, the analysis of such complex geometries becomes less problematic.

The stepping stone of this method is to enrich the standard finite element approximation space by a specific enrichment function $\psi$ which allows us to locate material interfaces and thus render the corresponding strains along these interfaces discontinuous. Following [32] and limiting attention to the elasticity the augmented approximation of the displacement field reads

$$
\boldsymbol{u}=\sum_{i \in I} N_{i} \boldsymbol{u}_{i}+\sum_{j \in I^{*}} N_{j}^{*} \psi \boldsymbol{a}_{j},
$$

where $N_{i}$ are the standard shape functions, $I$ represents the total number of finite element nodes in the analyzed domain, $I^{*} \subset I$ gives the number of nodes for which the support is split by the interface and $\boldsymbol{a}_{j}$ are the additional degrees of freedom. To properly capture the interface

\footnotetext{
3 Attention is limited to elasticity. The problem of heat and moisture transport is approached analogically by replacing the displacement field by the corresponding scalar quantities and the $6 \times 6$ stiffness matrix by the $3 \times 3$ moisture diffusivity or thermal conductivity matrices, see e.g. [15].
} 


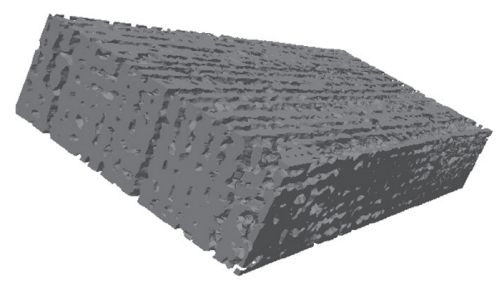

(a)

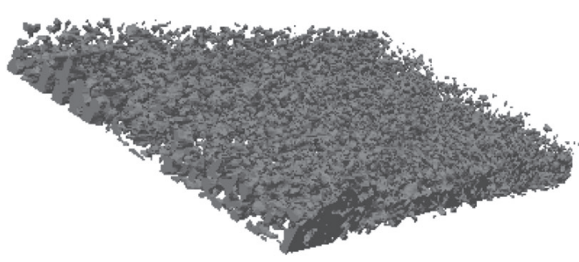

(b)

Figure 7: X-FEM representation of porosity: (a) Earlywood, (b) Latewood.

location within an element Sukumar et al. [33] applied a level set representation of surfaces through a level set function

$$
\phi^{\mathrm{LS}}(\boldsymbol{x})=\sum_{i \in J} N_{i}^{*}(\boldsymbol{x}) \phi_{i}^{\mathrm{LS}}
$$

where $N_{i}^{*}$ are the shape functions building a local partition of unity, in most cases $N_{i}^{*}=N_{i}$. Then, $J$ stands for the number of nodes of the element containing the point $\boldsymbol{x}$. The nodal values $\phi_{i}^{\mathrm{LS}}$ represent the signed distance of the element node to $i$ the interface with either a positive or a negative value depending on the material to which it belongs. This function then locates interfaces implicitly as a union of points for which it attains a zero value (zero-level). Given the level set function, Moës et al. [32] introduced a specific form of the enrichment function in the form

$$
\psi=\sum_{i \in J}\left|\phi_{i}^{\mathrm{LS}}\right| N_{i}^{*}-\left|\sum_{i \in J} \phi_{i}^{\mathrm{LS}} N_{i}^{*}\right|,
$$

where $\phi_{i}^{L S}$ denotes the level set value in the node $i$. Note that the actual value of $\psi$ along the interface is irrelevant as long as it captures the weak discontinuity in gradient fields properly. Owing to a space limitation we do not wish to elaborate this topic any further and instead refer the interest reader to $[32,15,31]$ for additional reading.

For illustration we plot the 3D X-FEM representation the porous phase for both the earlywood (Fig. 7(a)) and latewood (Fig. 7(a)) corresponding to the volume fractions stored in the 3rd row in Table 1. It is evident that the present level of resolution is highly insufficient as the actual shape of lumens seen in Figs. 1(b,c) is not properly captured. We further observe that in this case the porous phase is highly connected not only in the longitudinal direction, but also in the transverse directions. This will have a strong effect on the prediction of effective diffusivities in particular to be shown next in Section 4. To get a reliable image about 3D microstructure would thus call for much refined resolution deliverable for small samples using for example the computational nano-tomography [34].

\section{NUMERICAL EXAMPLES AND RESULTS}

Since the principal objective of this work is to capture the influence of porosity as good as possible, we start from the effective properties at the cell wall level listed in Table 2.

Their derivation based on the Mori-Tanaka method is available, e.g. in $[8,9,7,25] .{ }^{4}$ In the next homogenization step the values from Table 2 represent the homogenized matrix

\footnotetext{
4 The resulting elastic stiffnesses are found for dry wood, whereas the thermal conductivities and moisture diffusivities consider a specific moisture content $u=10 \%$ and temperature $\theta=20^{\circ} \mathrm{C}$. The latter predictions therefore account for the presents of water and other wood exctractives.
} 
Table 2: Effective material properties at the cell wall level.

\begin{tabular}{lclllc}
\hline \multicolumn{2}{c}{ Moduli [GPa $]$} & \multicolumn{2}{c}{ Conductivities $\left[\mathrm{Wm}^{-1} \mathrm{~K}^{-1}\right]$} & \multicolumn{2}{c}{ Diffusivities $\left[10^{-12} \mathrm{~m}^{2} \mathrm{~s}^{-1}\right]$} \\
$E_{L}$ & $E_{R}=E_{T}$ & $\lambda_{L}$ & $\lambda_{R}=\lambda_{T}$ & $D_{L}$ & $D_{R}=D_{T}$ \\
\hline 25.58 & 8.77 & 0.63 & 0.35 & 5.65 & 2.26 \\
\hline
\end{tabular}

properties of a porous material, the earlywood and latewood respectively. In most MT calculations the lumens are represented by an ellipsoid with semi-axes taken from [35]. ${ }^{5}$ The discrepancies in terms of phase volume fractions evident in Fig. 4(a) and Table 1 resulted in relatively large differences in the MT predictions at the level of wood as seen in Fig. 8. ${ }^{6}$

To address the issue of the volume fraction of pores further, we summarize in Tables 3-4 the predictions found at the level of earlywood and latewood from both the MT method and X-FEM. As for the Mori-Tanaka results, the analyses differ by the adopted volume fractions of pores and the shape of inclusions representing pores. The volume of pores is taken from 2D image analysis (IA), expressions based on dry wood density (Density), and computational micro-tomography $(\mu-\mathrm{CT})$, respectively, recall Table 1 . The $\mathrm{MT}^{E}(\mu-\mathrm{CT})$ and $\mathrm{MT}^{C}(\mu-\mathrm{CT})^{7}$ analyses were added to examine the influence of actual microstructure by comparing the MT predictions and estimates delivered by X-FEM, the last two rows in Tables 3-4.

It can be noticed that the adopted geometry of the elliptical cross-section, recall Fig. 1(b,c), of both ellipsoidal and cylindrical inclusions yielded, particularly in case of latewood, a relatively high anisotropy in MT predictions. ${ }^{8}$ However, this has not been confirmed by X-FEM calculations mostly attributed to a rather crude approximation of the actual 3D morphology of spruce wood used in this study, recall Fig. 7. While this has not proved to be so significant in the prediction of effective elastic moduli and thermal conductivities, see Tables 3 and 3, the opposite is true for moisture diffusivities evident from Table 5. Comparing the results

Table 3: Elastic moduli [GPa].

\begin{tabular}{lllllll}
\hline Method & \multicolumn{3}{c}{ Earlywood } & \multicolumn{3}{c}{ Latewood } \\
& $E_{L}$ & $E_{R}$ & $E_{T}$ & $E_{L}$ & $E_{R}$ & $E_{T}$ \\
\hline $\mathrm{MT}^{E}$ (IA) & 14.83 & 3.10 & 2.60 & 20.97 & 2.12 & 6.87 \\
$\mathrm{MT}^{E}$ (Density) & 5.62 & 0.88 & 0.69 & 13.55 & 0.64 & 4.14 \\
$\mathrm{MT}^{E}(\mu$-CT) & 6.39 & 1.02 & 0.81 & 20.46 & 1.92 & 6.67 \\
$\mathrm{MT}^{C}(\mu$-CT) & 6.39 & 1.02 & 0.81 & 20.46 & 1.92 & 6.67 \\
X-FEM & 3.78 & 0.52 & 0.21 & 18.89 & 5.61 & 5.12 \\
\hline
\end{tabular}

\footnotetext{
5 Recall Fig. 1 to see $a_{T}=14.85 \mu \mathrm{m}, a_{R}=18.15 \mu \mathrm{m}, a_{L}=1328.50 \mu \mathrm{m}$ for earlywood and $a_{T}=11.05 \mu \mathrm{m}, a_{R}=1.55 \mu \mathrm{m}$, $a_{L}=1325.00 \mu \mathrm{m}$ for latewood, respectively.

6 The results correspond to two additional homogenization steps at the level of lumens and annual rings. Point out that at the level of lumens we adopted eqn (2). In case of elasticity the $6 \times 6$ matrix $\mathbf{L}_{2}=\mathbf{0}$ was considered, whereas in case of properties the $3 \times 3$ matrix $\mathbf{L}_{2}$ was assumed diagonal with the diffusivity and thermal conductivity of the moist air equal to $D_{\text {air }}=2.6 \times 10^{-5} \mathrm{~m}^{2} \mathrm{~s}^{-1}$ and $\lambda_{\text {air }}=0.026 \mathrm{Wm}^{-1} \mathrm{~K}^{-1}$, respectively. The same properties were adopted also in the X-FEM analysis together with the cell wall properties derived from the MT method, see Table 2.

7 In Tables 3 and $4 \mathrm{MT}^{E}$ and $\mathrm{MT}^{C}$ stand for the application of ellipsoids and elliptic cylinders as representatives of the inclusion shape, respectively.

8 Point out that the ratio of semi-axis taken from [35] is about $1: 7$, which is much larger than the one derived from dry wood density amounting approximately to $1: 2$, see [16] for details.
} 


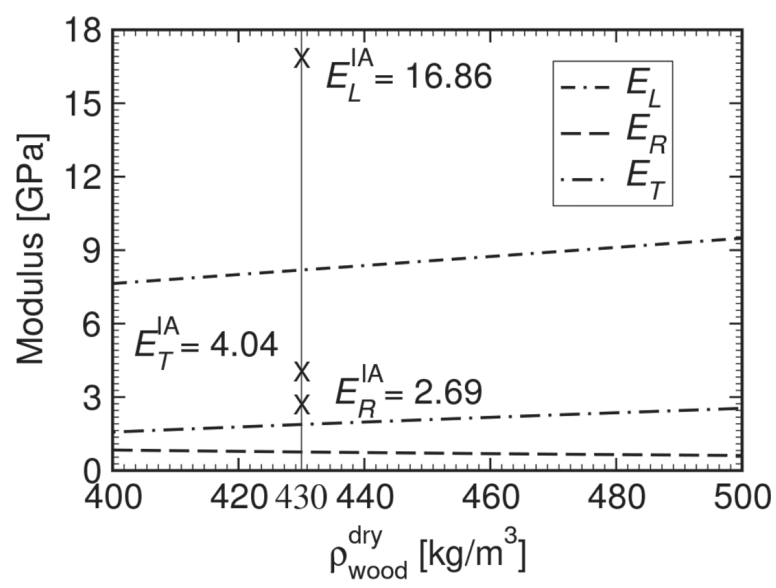

(a)

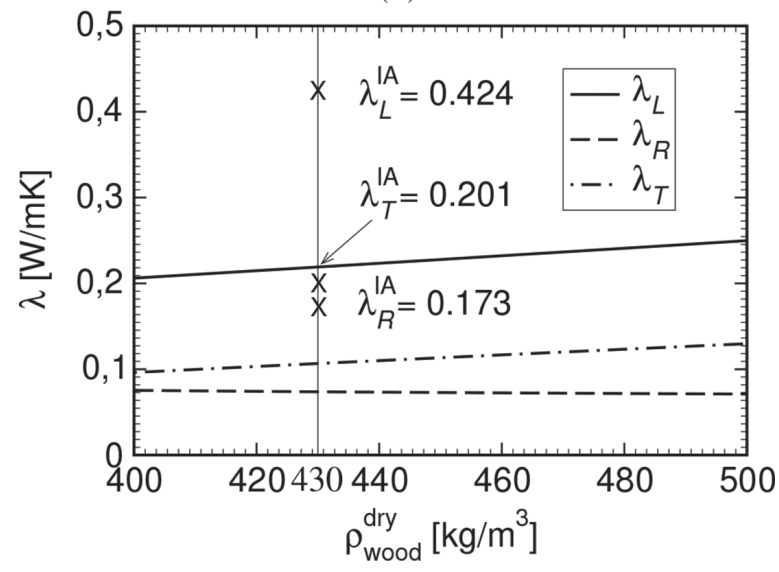

(b)

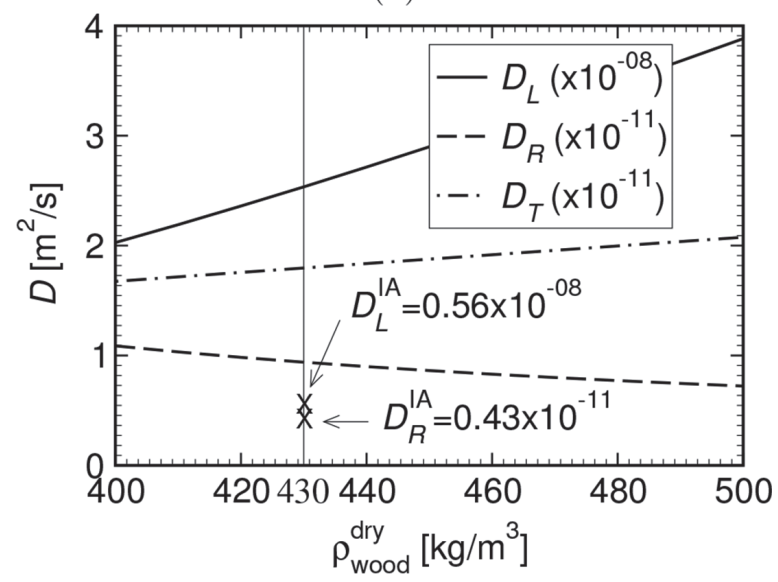

(c)

Figure 8: Mori-Tanaka estimates of the effective properties of wood: (a) elastic moduli, (b) thermal conductivities, (c) moisture diffusivities. 
Table 4: Thermal conductivities $\left[\mathrm{Wm}^{-1} \mathrm{~K}^{-1}\right]$.

\begin{tabular}{lllllll}
\hline Method & \multicolumn{3}{c}{ Earlywood } & \multicolumn{3}{c}{ Latewood } \\
& $\lambda_{L}$ & $\lambda_{\mathrm{R}}$ & $\lambda_{\mathrm{T}}$ & $\lambda_{L}$ & $\lambda_{\mathrm{R}}$ & $\lambda_{\mathrm{T}}$ \\
\hline $\mathrm{MT}^{E}$ (IA) & 0.38 & 0.17 & 0.16 & 0.52 & 0.18 & 0.29 \\
$\mathrm{MT}^{E}$ (Density) & 0.15 & 0.07 & 0.06 & 0.35 & 0.08 & 0.19 \\
$\mathrm{MT}^{E}(\mu-\mathrm{CT})$ & 0.18 & 0.08 & 0.07 & 0.51 & 0.17 & 0.28 \\
$\mathrm{MT}^{C}(\mu$-CT) & 0.18 & 0.08 & 0.07 & 0.51 & 0.17 & 0.28 \\
$\mathrm{X}-\mathrm{FEM}$ & 0.15 & 0.06 & 0.05 & 0.49 & 0.25 & 0.24 \\
\hline
\end{tabular}

Table 5: Moisture diffusivities $\left[10^{-12} \mathrm{~m}^{2} \mathrm{~s}^{-1}\right]$

\begin{tabular}{llccccc}
\hline Method & \multicolumn{3}{c}{ Earlywood } & \multicolumn{3}{c}{ Latewood } \\
& $D_{L}$ & $D_{R}$ & $D_{T}$ & $D_{L}$ & $D_{R}$ & $D_{T}$ \\
\hline $\mathrm{MT}^{E}(\mathrm{IA})$ & $0.3 \times 10^{4}$ & 6 & 5 & $1.1 \times 10^{4}$ & 3 & 6 \\
$\mathrm{MT}^{E}$ (Density) & $1.5 \times 10^{4}$ & 18 & 17 & $4.5 \times 10^{4}$ & 5 & 19 \\
$\mathrm{MT}^{E}(\mu$-CT) & $1.2 \times 10^{4}$ & 17 & 15 & $1.3 \times 10^{4}$ & 3 & 7 \\
$\mathrm{MT}^{C}(\mu$-CT) & $1950 \times 10^{4}$ & 17 & 15 & $519 \times 10^{4}$ & 3 & 7 \\
X-FEM & $1869 \times 10^{4}$ & $1604 \times 10^{4}$ & $1304 \times 10^{4}$ & $378 \times 10^{4}$ & $65 \times 10^{4}$ & $86 \times 10^{4}$ \\
\hline
\end{tabular}

obtained by the MT method suggests high dependency of predictions of effective properties on the actual shape of inclusions representing pores:

- Even a relatively large difference in volume fractions of pores for earlywood and latewood may eventually result in comparable values of effective properties depending on the shape of the inclusion cross-section, Figs. 1(b,c). For some combinations of phase porosity the effective properties of latewood may even exceed those for the earlywood, see the 2nd row in Table 5.

- An important role plays also the selected type of inclusion no so evident for the case of elasticity and thermal conductivity. Despite the fact that the major axis of the ellipsoid is relatively large compare to its minor axes, recall the footnote No. 5, the assumption of this axis being infinite, as in case of elliptic cylinder, renders of the order of magnitude larger values of longitudinal diffusivities, compare the 3rd and 4th row in Table 5. Interestingly, these values are similar to the X-FEM results. This may prefer the use of cylindrical inclusions to ellipsoids as the former ones may better account for the presence of pits, interconnection of lumens.

\section{SUMMARY AND CONCLUSIONS}

Several computational approaches to the prediction of effective properties of wood were examined in the light of both mechanical and non-mechanical load sources. The results provided by the application of the Mori-Tanaka method only and the results derived by combining the Mori-Tanaka method at the level of cell wall and the XFEM calculations performed on a suitable RVE on mesoscale were compared. The following, rather qualitative conclusions, can be drawn: 
- Prediction of effective properties is highly influenced by the assumed porosity, the volume fraction of lumens. This is not an easy task. A quick estimate is available based on the dry wood density [16] with some improvements proposed in [5]. More reliable approach was expected from the application of computational micro-tomography. Unfortunately, the present measurements proved insufficient and call for improvements.

- In case of the Mori-Tanaka method the properly chosen type and shape of the inclusion representing lumens plays a crucial role particularly when searching for effective moisture diffusivities. Such a statement has recently been supported by the results presented in $[5,36]$.

- To allow also for reliable quantitative conclusions requires a better formulation of RVE for numerical analysis and experimentally measured values of effective properties. Both issues are being currently addressed and the results will be presented elsewhere. Some initiatives can be found in $[11,5,36]$.

- Attention also deserves prediction of the microfibril angle from nanoindentation measurements recently discussed in [36] with attention to the estimation of experimental error with the help of Bayesian inference. Such an error can be significant and may have a considerable effect on the final prediction of the searched effective properties.

\section{ACKNOWLEDGMENTS}

The financial support provided by the GAČR grant No. 18-05791S is gratefully acknowledged.

\section{REFERENCES}

[1] Ormarsson, S. \& Dahlblom, O., Finite element modelling of moisture related and visco-elastic deformations in inhomogeneous timber beams. Engineering Structures, 49, pp. 182-89, 2013. https://doi.org/10.1016/j.engstruct.2012.10.019

[2] Fortino, S., Mirianon, F. \& Toratti, T., A 3d moisture-stress FEM analysis for time dependent problems in timber structures. Mechanical and Time-Dependendent Materials, 13(4), pp. 333-56, 2009. https://doi.org/10.1007/s11043-009-9103-z

[3] Dang, D., Moutou Pitti, R., Toussaint, E. \& Grédiac, M., Investigating wood under thermohydromechanical loading at the ring scale using full-field measurements. Wood Science and Technology, 52(6), pp. 1473-93, 2018. https://doi.org/10.1007/s00226-018-1051-9

[4] Rafsanjani, A., Lanvermann, C., Niemz, P., Carmeliet, J. \& Derome, D., Musltiscale analysis of free swelling of Norway spruce. Composites: Part A, 54, pp. 70-78, 2013. https://doi.org/10.1016/j.compositesa.2013.07.005

[5] Šejnoha, M., Sýkora, J., Vorel, J., Kucíková, L., Antoš, J., Pokorný, J. \& Pavlík, Z., Moisture induced strains in spruce from homogenization and transient moisture transport analysis. Computers and Structures, 2018. Submitted.

[6] Melzerová, L., Kucíková, L., Janda, T. \& Šejnoha, M., Estimation of orthotropic mechanical properties of wood based on non-destructive testing. Wood Research, 61(6), pp. 861-70, 2016.

[7] Šejnoha, M., Janda, T., Melzerová, L., Nežerka, V. \& Šejnoha, J., Modeling glulams in linear range with parameters updated using Bayesian inference. Engineering Structures, 138, pp. 293-307, 2017. https://doi.org/10.1016/j.engstruct.2017.02.021

[8] Šejnoha, M., Kucíková, L., Vorel, J., Hrbek, V. \& Němeček, J., Comparing nano and macroindentation in search for microfibril angle in spruce. Internationl Journal of Computational Methods and Experimental Measurements, 5(2), pp. 135-43, 2017. https://doi.org/10.2495/cmem-v5-n2-135-143 
[9] Šejnoha, M., Janda, T., Vorel, J., Kucíková, L. \& Padevěd, P., Combining homogenization, indentation and bayesian inference in estimating the microfibril angle of spruce. Procedia Engineering, 190, pp. 310-17, 2017. https://doi.org/10.1016/j.proeng.2017.05.343

[10] Jäger, A., Bader, T., Hofstetter, K. \& Eberhardsteiner, J., The relation between indentation modulus, microfibril angle, and elastic properties of wood cell walls. Composites Part A: Applied Science and Manufacturing, 42(6), pp. 677-85, 2011. https://doi. org/10.1016/j.compositesa.2011.02.007

[11] Persson, K., Micromechanical Modelling of Wood and Fibre Properties. Ph.D. thesis, Publ. TVSM-1013, Div. of Struc. Mech., Lund University, 2000.

[12] Eitelberger, J. \& Hofstetter, K., Prediction of transport properties of wood below the fiber saturation point-a multiscale homogenization approach and its experimental validation: Part I: thermal conductivity. Composites Science and Technology, 71(2), pp. 134-44, 2011. https://doi.org/10.1016/j.compscitech.2010.11.007

[13] Eitelberger, J. \& Hofstetter, K., Prediction of transport properties of wood below the fiber saturation point a multiscale homogenization approach and its experimental validation. part II: Steady state moisture diffusion coefficient. Composites Science and Technology, 71(2), pp. 145-51, 2011. https://doi.org/10.1016/j.compscitech.2010.11.006

[14] Benveniste, Y., A new approach to the application of Mori-Tanaka theory in composite materials. Mechanics of Materials, 6(2), pp. 147-57, 1987. https://doi.org/10.1016/01676636(87)90005-6

[15] Šejnoha, M. \& Zeman, J., Micromechanics in Practice. WIT Press, Southampton, Boston, 2013.

[16] Hofstetter, K., Hellmich, C. \& Eberhardsteiner, J., Development and experimental validation of a continuum micromechanics model for the elasticity of wood. European Journal of Mechanics -A/Solids, 24(6), pp. 1030-1053, 2005. https://doi.org/10.1016/j. euromechsol.2005.05.006

[17] Vorel, J., Sýkora, J., Urbanova, S. \& Šejnoha, M., From CT scans of wood to_nite element meshes. Proceedings of the The Fifteenth International Conferenceon Civil, Structural and Environmental Engineering Computing, Prague, CzechRepublic, ed. B. Topping, Civil-Comp Press, 2015. https://doi.org/10.4203/ccp.108.221

[18] Tze, W.T.Y., Wang, S., Rials, T.G., Pharr, G.M. \& Kelley, S.S., Nanoindentation of wood cell walls: Continuous stiffness and hardness measurements. Composites: Part A, 38(3), pp. 945-53, 2007. https://doi.org/10.1016/j.compositesa.2006.06.018

[19] Gamstedt, E.K., Bader, T.K. \& de Borst, K., Mixed numerical-experimental methods in wood micromechanics. Wood Science and Technology, 47(1), pp. 183-202, 2013. https://doi.org/10.1007/s00226-012-0519-2

[20] Vlassak, J. \& Nix, W., Measuring the elastic properties of anisotropic materials by means of indentation experiments. Journal of the Mechanics and Physics of Solids, 42(8), pp. 1223-45, 1994. https://doi.org/10.1016/0022-5096(94)90033-7

[21] Vlassak, J., Ciavarella, M., Barber, J. \& Wang, X., The indentation modulus of elastically anisotropic materials for indenters of arbitrary shape. Journal of the Mechanics and Physics of Solids, 51(9), pp. 1701-21, 2003. https://doi.org/10.1016/0022-5096(94)90033-7

[22] Arifin, A.Z. \& Asano, A., Image segmentation by histogarm thresholding using hierarchical cluster analysis. Pattern Recognition Letters, 27, pp. 1515-21, 2006. https://doi. org/10.1016/j.patrec.2006.02.022

[23] Fíla, T., Kumpová, I., Koudelka, P., Zlámal, P., Vavř́ík, D., Jiroušek, O. \& Jung, C., Dual-energy x-ray micro-ct imaging of hybrid Ni/Al. 7th International Workshop on 
Radiation Imaging Detectors 28 June-2 July 2015, DESY, Hamburg, Germany, 2015. https://doi.org/10.1088/1748-0221/11/01/c01005

[24] Benveniste, Y., Dvorak, G.J. \& Chen, T., On diagonal and elastic symmetry of the approximate effective stiffness tensor of heterogeneous media. Journal of the Mechanics and Physics of Solids, 39(7), pp. 927-46, 1991. https://doi.org/10.1016/ 0022-5096(91)90012-d

[25] Kucíková, L., Vorel, J., Sýkora, J. \& Šejnoha, M., Effective heat and moisture transport properties of spruce. 23rd International conference Engineering Mechanics 2017, 2017.

[26] Teplý, J.L. \& Dvorak, G.J., Bound on overall instantaneous properties of elastic-plastic composites. Journal of the Mechanics and Physics of Solids, 36(1), pp. 29-58, 1988. https://doi.org/10.1016/0022-5096(88)90019-1

[27] Michel, J.C., Moulinec, H. \& Suquet, P., Effective properties of composite materials with periodic microstructure: A computational approach. Computer Methods in Applied Mechanics and Engineering, 172(1-4), pp. 109-143, 1999. https://doi.org/10.1016/ s0045-7825(98)00227-8

[28] Fish, J. \& Shek, K., Multiscale analysis of large-scale nonlinear structures and materials. International Journal for Computational Civil and Structural Engineering, 1(1), pp. 79-90, 2000.

[29] Kouznetsova, V., Geers, M.G.D. \& Brekelmans, W.A.M., Multi-scale constitutive modelling of heterogeneous materials with a gradient-enhanced computational homogenization scheme. International Journal for Numerical Methods in Engineering, 54(8), pp. 1235-1260, 2002. https://doi.org/10.1002/nme.541

[30] Zeman, J. \& Šejnoha, M., From random microstructures to representative volume elements. Modelling and Simulation in Materials Science and Engineering, 15(4), pp. S325-S335, 2007. https://doi.org/10.1088/0965-0393/15/4/s01

[31] Vorel, J., Grippon, E. \& Šejnoha, M., Effective thermoelastic properties of polysiloxane matrix based plain weave textile composites. International Journal for Multiscale Computational Engineering, 13(3), pp. 181-200, 2015. https://doi.org/10.1615/intjmultcompeng.2014011020

[32] Moës, N., Cloirec, M., Cartraud, P. \& Remacle, J.F., A computational approach to handle complex microstructure geometries. Computer Methods in Applied Mechanics and Engineering, 192(28-30), pp. 3163-77, 2003. https://doi.org/10.1016/s00457825(03)00346-3

[33] Sukumar, N., Chopp, D.L., Moës, N. \& Belytschko, T., Modeling holes and inclusions by level sets in the extened finite element method. Computer Methods in Applied Mechanics and Engineering, 190(46-47), pp. 6183-200, 2001. https://doi.org/10.1016/ s0045-7825(01)00215-8

[34] Vaziri, M., du Plessis, A., Sandberg, D. \& Berg, S., Nano x-ray tomography analysis of the cell-wall density of welded beech joints. Wood Material Science and Engineering, 2015. https://doi.org/10.1080/17480272.2015.1062418

[35] Kettunen, P.O., Wood: Structure and Properties. Trans Tech Publications Ltd, UetikonZuerich, 2006.

[36] Šejnoha, M., Janda, T., Vorel, J., Kucíková, L., Padevět, P. \& Hrbek, V., Bayesian inference as a tool for improving predictions of effective elastic properties of wood. Computers and Structures, 2018. Submitted. 